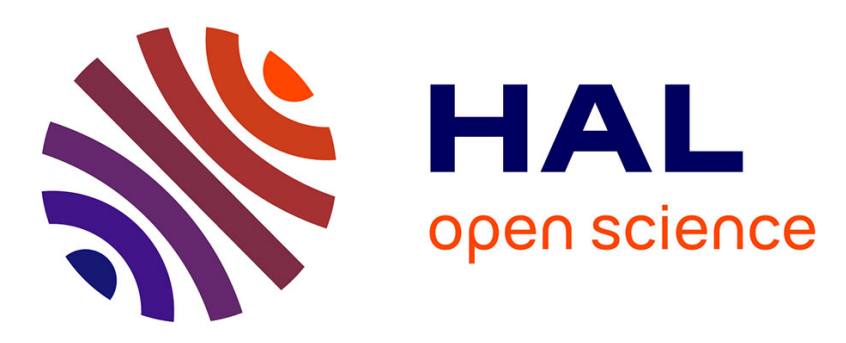

\title{
A new method to assess weight-bearing distribution after central nervous system lesions in rats
}

Vincent Pertici, Caroline Pin-Barre, Marie-Solenne Felix, Jérôme Laurin, Jeanick Brisswalter, Patrick Decherchi

\section{- To cite this version:}

Vincent Pertici, Caroline Pin-Barre, Marie-Solenne Felix, Jérôme Laurin, Jeanick Brisswalter, et al.. A new method to assess weight-bearing distribution after central nervous system lesions in rats. Behavioural Brain Research, 2014, 259, pp.78 - 84. 10.1016/j.bbr.2013.10.043 . hal-01475744

\author{
HAL Id: hal-01475744 \\ https://hal.science/hal-01475744
}

Submitted on 26 Feb 2017

HAL is a multi-disciplinary open access archive for the deposit and dissemination of scientific research documents, whether they are published or not. The documents may come from teaching and research institutions in France or abroad, or from public or private research centers.
L'archive ouverte pluridisciplinaire HAL, est destinée au dépôt et à la diffusion de documents scientifiques de niveau recherche, publiés ou non, émanant des établissements d'enseignement et de recherche français ou étrangers, des laboratoires publics ou privés. 


\title{
A new method to assess weight-bearing distribution after central nervous system lesions in rats.
}

\author{
Vincent PERTICI ${ }^{* 1}$, Caroline PIN-BARRE*2 ${ }^{* 2}$, Marie-Solenne FELIX ${ }^{1}$, Jérôme \\ LAURIN $^{\bowtie 1}$, Jeanick BRISSWALTER ${ }^{2}$, Patrick DECHERCHI ${ }^{1}$
}

*These authors contributed equally to this work.

${ }^{1}$ Aix-Marseille Université (AMU) et Centre National de la Recherche Scientifique (CNRS)

UMR 7287 «Institut des Sciences du Mouvement (ISM) : Etienne-Jules MAREY »

Equipe «Plasticité des Systèmes Nerveux et Musculaire » (PSNM)

Parc Scientifique et Technologique de Luminy

Faculté des Sciences du Sport de Marseille

CC910 - 163 Avenue de Luminy

F-13288 Marseille cedex 09, France

${ }^{2}$ Université de Nice Sophia-Antipolis et Université du Sud Toulon Var

EA 6309 « Motricité Humaine Éducation Sport Santé » (LAMHESS)

UFR STAPS de Nice

261, route de Grenoble

F-06205 Nice, France

\author{
Corresponding author: \\ ${ }^{\triangle}$ Dr Jérôme LAURIN \\ UMR 7287 «Institut des Sciences du Mouvement (ISM) : Etienne-Jules MAREY » \\ Equipe « Plasticité des Systèmes Nerveux et Musculaire » (PSNM) \\ Aix-Marseille Université (AMU) et Centre National de la Recherche Scientifique (CNRS) \\ Faculté des Sciences du Sport de Marseille \\ CC910 - 163, avenue de Luminy \\ 13288 Marseille cedex 09 - France \\ E-mail: jerome.laurin@univ-amu.fr \\ Tel.: +33 (0)4-91-82-84-11 \\ Web site: $w w w . i s m . u n i v m e d . f r$
}

Short title: Functional assessment after CNS Alteration. 


\begin{abstract}
The aim of the present study is to assess the relevance of weight-bearing distribution (DWB) measurement in freely moving rats after stroke and thoracic spinal cord injuries. Animals were divided in 2 experiments: 1) The middle cerebral artery occlusion-reperfusion (MCAO-r) experiment containing the MCAO group in which focal brain ischemia was induced by transient MCA occlusion and 2) the thoracic hemisection experiment containing the $\mathrm{TH}$ group in which a spinal cord hemisection was performed at the $\mathrm{T}_{10}$ level. A Control and respective Sham groups were also included in each experiment. Not only the pressure exerted by each paw was measured but also different ratios such as: the sum of the right and the left forepaws was normalized by the sum of the right and the left hindpaws $(\mathrm{F} / \mathrm{H}), 2)$ the left forepaw was normalized by the right forepaw (LF/RF), 3) the left hindpaw was normalized by the right hindpaw (LH/RH). Additionally, the times spent on 3 paws and on 4 paws were measured. Only the time spent on 4 paws was shorter in the MCAO group than in the Control $(p<0.001)$ and in the Sham $(p<0.01)$ groups. The LH/RH ratio of the TH group at the $1^{\text {st }}$ week was lower $(\mathrm{p}<0.01)$ than the pre-surgical value. Moreover, its $\mathrm{F} / \mathrm{H}$ ratio was superior $(p<0.001)$ to the ones of the Control and the Sham groups. Our study indicates that DWB should be more frequently used to evaluate both the severity of central nervous system traumas and the effectiveness of pharmacological and/or rehabilitation strategies.
\end{abstract}

Key words: Stroke, Spinal cord injury, postural strategy, neuromuscular plasticity. 


\section{Introduction}

Brain stroke and spinal cord injuries (SCI) are the leading reasons for long termdisability. More precisely, these pathologies are characterized by motor, sensory and autonomic dysfunctions $[1,2]$. Due to the fact that restoration of behavioural functions for patient is the main goal, efficient identification of behavioural changes is a prerequisite for translational applications [3]. Behavioural assessment on rodents is essential to explore the extent of functional deficits and the beneficial effects of treatments after such traumas.

Currently, a wide range of behavioural tests has already been approved to quantify the motor and sensory residual functions after spinal cord injuries $[4,5]$ or brain stroke $[3,6]$. Whatever the type of behavioural assessment (sensorimotor or locomotor tests), it was considered that a functional test should preferentially avoid familiarization procedure, fatigue accumulation, complex tasks, stress, food deprivation and motivation dependence [7].

The dynamic weight-bearing test (DWB), assessing the distribution of the pressure exerted by each paw, is exempt from the above-cited drawbacks. Indeed, contrary to the preceding static weight-bearing test (SWB) carried out on restrained rats that only analysed the hindpaws pressure, the DWB test is performed on free moving rats (hence the term "dynamic"). Thus, each paw pressure can be analysed during static position periods, while avoiding stress accumulation. Moreover, the computer-assisted DWB test produces objective data allowing reproducibility which is also recommended for a behavioural test [8]. Consequently, the DWB test seems appropriate to reveal impairments induced by CNS injuries and to determine difference between the affected limbs. However, no information indicated that the DWB test could be used to detect behavioural alterations after CNS traumas.

The present study is designed to evaluate the relevance of weight-bearing distribution measurement in freely moving rats after CNS trauma (SCI and stroke). Because there are specific time periods for detecting impairments in each CNS trauma, we have chosen different recording time period depending on the lesion type. Stroke induces major physiological modifications during the $1^{\text {st }}$ week post-ischemia $[9,10]$. By contrast, a longer time period of at least 6 weeks was required for thoracic spinal cord hemisection (TH) [11]. 


\section{Materials and methods}

\subsection{Animals.}

Thirty-nine adult male Sprague Dawley rats, weighting 250-300g (Centre d'Elevage Roger JANVIER ${ }^{\circledR}$, Le Genest Saint Isle, France), were housed in smooth-bottomed plastic cages at $22{ }^{\circ} \mathrm{C}$ with a 12-h light/dark cycle. Food (Safe ${ }^{\circledR}$, Augy, France) and water were available ad libitum. An acclimation period of 1 week was allowed before the initiation of the experiment. The weight of the rats was daily controlled.

The present study gathered 2 experiments: 1) The middle cerebral artery occlusionreperfusion (MCAO-r) experiment containing the MCAO group $(n=8)$ in which focal brain ischemia was induced by transient MCA occlusion and the SHAM group $(n=6)$ in which the surgery was performed without the occlusion and 2) the thoracic hemisection experiment containing the TH group $(n=7)$ in which a spinal cord hemisection was performed at the $\mathrm{T}_{10}$ level and the SHAM group $(n=6)$ in which the surgery was carried out without the spinal cord hemisection. Additionally, a Control group $(n=12)$ was also included in the study.

\subsection{Ethical approval.}

Anaesthesia and surgical procedures were performed according to the French law on animal care guidelines and the Animal Care Committees of Aix-Marseille Université (AMU) and Centre National de la Recherche Scientifique (CNRS) approved our protocols. Individual conducting researches were listed in the authorized personnel section of the animal research protocol or added to a previously approved protocol. Furthermore, experiments were performed following the recommendations provided in the Guide for Care and Use of Laboratory Animals (U.S. Department of Health and Human Services, National Institutes of Health) and in accordance with the European Community's council directive of 24 November 1986 (86/609/ EEC).

No clinical sign of pain or unpleasant sensation (i.e. screech, prostration, hyperactivity, anorexia) and no paw-eating behaviour were observed through the study.

At the end of the experiments, animals were sacrificed by an intraperitoneal overdose $(3 \mathrm{ml})$ of sodium pentobarbital solution $\left(\mathrm{Nembutal}^{\circledR}\right.$, Sanofi Santé Animale, Libourne, France -0.6 g. $\left.\mathrm{kg}^{-1}\right)$.

\subsection{Surgical protocols.}

\subsubsection{Middle Cerebral Artery Occlusion-Reperfusion.}

In order to optimize reproducibility of the cerebral lesion severity, it is noteworthy to precise that the MCAO-r was performed on rat weighting exclusively between $250 \mathrm{~g}$ and 270 
g. Animals weighting more than $270 \mathrm{~g}$ were excluded from the study. Central temperature was maintained at about $37-38^{\circ} \mathrm{C}$ with a homeothermic blanket (Harvard Homeothermic Blanket Control Unit, K01345CE) driven by a rectal thermal probe.

Anaesthesia was induced with 5\% isoflurane and maintained with 2.5-2 \% isoflurane through a facemask, supplemented with oxygen. A $0.2 \mathrm{ml}$ injection of $0.5 \%$ bupivacaine was subcutaneously performed along the prospective incision site. Throughout the surgery, animals were positioned in dorsal decubitus. A $4 \mathrm{~cm}$ ventral midline incision was performed and the superficial fascia was dissected. The right external, internal, and common carotid arteries (ECA, ICA, CCA) were exposed without damaging the vagus nerve and its collaterals. The occipital artery (OA) and the superior thyroid artery (TS) were cauterized (Bovi Medical Cooporation Clearwater, FL 33760, USA). After placing microsurgical clips on CCA and ICA, a partial arteriotomy was performed on ECA with micro-scissors. A 4-0 monofilament nylon suture (total length: $3 \mathrm{~cm}$; silicon-coated tip length and diameter: $5 \mathrm{~mm}$ and 0.39 $\pm 0.02 \mathrm{~mm}$ respectively; Doccol Corp, MCAO suture PK10, 40-333PK10, Redland, CA, USA) was inserted into the ECA lumen down toward the CCA where the microsurgical clip was located. The tip of the monofilament was then introduced into the ICA via the ECA and approximately pushed $20 \mathrm{~mm}$ away from the carotid bifurcation. Blood flow was thus blocked at the MCA origin. After 120 min of occlusion, the monofilament was carefully removed and the ICA was permanently closed by electrocoagulation to prevent bleeding [12, 13]. Finally, the skin was sutured and animals returned in individual cages.

\subsubsection{Thoracic spinal hemisection.}

Rats were anaesthetized with choral hydrate intraperitoneal injection $\left(0.5 \mathrm{~g} . \mathrm{kg}^{-1}\right.$, Sigma, St. Louis, MO, USA). The animal's back was shaved and aseptically prepared with a povidone-iodine scrub swab stick (Professional Disposables, Orangeburg, NY). Central temperature was maintained constant at $37-38^{\circ} \mathrm{C}$ with a homeothermic blanket (Harvard Apparatus $^{\circledR}$, Holliston, USA) driven by a rectal thermal probe. With the aid of a dissecting microscope, a midline incision was made over the $\mathrm{C}_{6}-\mathrm{T}_{13}$ spinous processes. Dorsal muscles were cut and maintained on the side using retractors. While firmly maintaining the spinal column by spinous processes, a laminectomy at the $\mathrm{T}_{9}-\mathrm{T}_{10}$ vertebral levels was performed using a Friedman-Pearson Rongeur (0.7 mm Cup Curved, FST, Heidelberg, Germany). Following dura incision, the exposed dorsal surface of the spinal cord was rinsed with cold saline to favour vasoconstriction. Finally, a left spinal cord hemi-transection was realized by the use of a sharp blade. Close examination of the cut edges of the cord confirmed that the surface of the tissue was free of meninges or blood clots. The lesion site was covered with a film of bioabsorbable artificial dural substitute (Seamdura ${ }^{\circledR}$, Gunze Ltd., Codman, Johnson 
and Johnson Company) to isolate spinal cord from the overlying muscles. Muscles and skin were separately sutured (Vicryl ${ }^{\circledR} 3-0$, Ethicon France). Rats were rehydrated with a bolus of saline ( $3 \mathrm{ml}$, subcutaneous) to replace fluid lost during the surgical procedure and received a subcutaneous injection of an antalgic (semi-synthetic opioid, buprenorphine, $0.05 \mathrm{mg} \mathrm{kg}^{-1}$ ). Then, they were kept in individual cages under heat lamps for 12-24 h. Housed in a nonenriched environment, the rats were daily observed during the next 8 weeks. Manual bladder expression was performed at least twice daily until bladder reflex was re-established.

\subsection{Dynamic weight-bearing distribution assessment}

The weight-bearing distribution was assessed by a biometric floor instrumented cage (Dynamic Weight-bearing, Bioseb ${ }^{\circledR}$ Development, Vitrolles, France)(Figure 1). The device consisted of a Plexiglas enclosure (W22 ${ }^{\circledast}$ L22 $\times \mathrm{H} 30 \mathrm{~cm}^{3}$ ) with a calibrated weight transducer pad composed of 44 x 44 captors (Captor surface: $10.89 \mathrm{~mm}^{2}$; Captor threshold: $0.1 \mathrm{~g}$; Matrix Sensor 5250 type: /10, TEKSCAN Inc. Boston, MA, USA). The rat was allowed to move freely within the apparatus for 2 trials of 5 min each. Using a synchronized videorecording and a scaled map of the stimulated captors, each paw of the rat was validated by an observer and identified as a unique paw. Only stable paw pressures of at least $0.2 \mathrm{sec}$ were kept for further analysis.

The pressure exerted by paw. The pressure exerted by each paw (in grams) was only measured when the four paws were in contact with the biometric floor and then normalized by the total weight of the rat. Ratios distinguishing the forepaws vs. hindpaws and the right vs. left side were calculated to assess the weight-bearing distribution: 1) the sum of the right and the left forepaws (F) was normalized by the sum of the right and the left hindpaws $(\mathrm{H})(\mathrm{F} / \mathrm{H}$ ratio), 2) the left forepaw (LF) was normalized by the right forepaw (RF) (LF/RF ratio), 3) the left hindpaw $(\mathrm{LH})$ was normalized by the right hindpaw $(\mathrm{RH})(\mathrm{LH} / \mathrm{RH}$ ratio).

Time spent on 3 paws and on 4 paws. The time spent on 3 paws and on 4 paws (in seconds) was measured to determine the solicitation of the affected paw in postural stability. In other words, if the affected paw was not used in static position, the time spent on 3 paws should be longer. The time spent on 2 paws referring to the explorative period was discarded for analysis. Given that the software just kept static position of the rat, the recording period could vary between animals. Consequently, the time periods spent on 3 paws and on 4 paws were normalized by the total time spent in static position for each recording.

\subsection{Neurological examination after MCAO-r.}


A neurological examination was performed after MCAO-r to ensure that the ischemia induced reliable sensorimotor impairments. A neurologic grading system with a five-point scale (0-4) was used: $0=$ no apparent deficits; $1=$ left forepaw flexion; $2=$ decreased grip of the left forepaw while tail pulled; 3 = spontaneous movement in all directions; left circling only if pulled by the tail; $4=$ spontaneous left circling [14].

\subsection{Basso, Beattie, and Bresnahan Scale Locomotor Assessment (BBB score)} after $\mathbf{T}_{10}$ hemisection.

The BBB score was used to ensure that the thoracic spinal cord injury induced significant locomotor deficits [15]. The BBB score assesses hindlimbs functions on a scale from 0 (complete hindlimb paralysis) to 21 (normal locomotion). Each point represents a specific set of characteristics displayed by the animals during free-moving locomotion. The test was performed in an open field once a week for 8 weeks. This test takes into account the motion degree of particular joints of the hindlimbs, the trunk stability during movement and the placement of each paw of the hindlimbs.

\subsection{Statistical analysis}

Data processing was performed using a software program (SigmaStat ${ }^{\circledR} 2.03$, Statistical software, San Jose, CA, USA). Data were expressed as mean \pm SEM.

The pressures exerted by each paw in percent of the total weight of the rat were averaged and compared in pre-surgical and post-surgical sessions. In order to determine differences between groups over time, a two-way repeated measures ANOVA (group x time) was calculated. Post-hoc multiple group comparisons were performed with a StudentNewman-Keuls Method.

Results were considered significant if the p-value fell below 0.05 . 


\section{Results}

\subsection{Cerebral ischemia.}

The rat weight decreased between $11 \%$ and 20\% after MCAO-r throughout protocol duration while the weight of SHAM rats was similar to the one of Control rats.

\subsubsection{Neurological examination after MCAO-r.}

No significant difference was observed between Control and SHAM groups. The neurological score of the MCAO group was significantly increase compared to both Control and SHAM groups from the $1^{\text {st }}$ to the $7^{\text {th }}$ day $(\mathrm{p}<0.001$; score $=4$ at day 1 and day 2 . Then, the score $=3$ from day 3 to day 7$)$ (Table 1$)$.

\subsubsection{The dynamic weight-bearing test after MCAO-r.}

Pressure exerted by each paw and pressure ratios. No significant difference was observed in the pressure (in grams) exerted by each paw and in the pressure ratios within and between groups (data not shown).

Time spent on 4 paws. Given that results of time spent on 3 paws followed the exact opposite behavioural pattern compared to results of time spent on 4 paws, only data of the latter were presented.

No difference was observed in the Control and the SHAM groups. Likewise, no difference was also found between these 2 groups. The pre-surgical time spent on 4 paws was similar between all groups. The time spent on 4 paws (normalized by total time) was significantly shorter in the MCAO group than in the Control $(p<0.001)$ and in the SHAM $(\mathrm{p}<0.01)$ groups. Moreover, the times spent on 4 paws in the MCAO group at the $2^{\text {nd }}$ $(\mathrm{p}<0.05), 6^{\text {th }}$ and $7^{\text {th }}($ both $\mathrm{p}<0.001)$ days were significantly shorter than the pre-surgical values. The times spent on all fours from the $1^{\text {st }}$ to the $7^{\text {th }}$ day in the MCAO group were significantly lower $(\mathrm{p}<0.01 ; \mathrm{p}<0.01 ; \mathrm{p}<0.05 ; \mathrm{p}<0.05 ; \mathrm{p}<0.01 ; \mathrm{p}<0.001$ and $\mathrm{p}<0.001$, respectively) than the ones of the Control group. The times spent on 4 paws at the $2^{\text {nd }}, 6^{\text {th }}$ and $7^{\text {th }}$ days post-surgery in the MCAO group were significantly $(p<0.05 ; p<0.001$ and $p<0.001)$ lower than the ones of the SHAM group. The time spent on 4 paws at the $5^{\text {th }}$ day in the SHAM group was higher than in the Control group $(\mathrm{p}<0.05)$ (Figure 2).

\subsection{Spinal cord injury.}

\subsubsection{The BBB score after $T_{10}$ hemisection.}

One week after the lesion, the BBB score in the TH group $(2.43 \pm 0.95)$ was significantly lower $(p<0.001)$ than the one of the Control and SHAM groups $(21.00 \pm 0.00$ for 
both). The BBB score in the TH group stayed significantly lower $(\mathrm{p}<0.001)$ than the 2 other groups through the protocol (Figure 3).

\subsubsection{The dynamic weight-bearing test after $T_{10}$ hemisection.}

Pressure exerted by each paw. No difference was observed in the Control and the SHAM groups. Likewise, no difference was also found between these 2 groups. Moreover, pre-surgical data of all the characteristics were similar between the 3 groups.

The pressures exerted by the LF of the TH group were significantly higher than the ones of the Control $(p<0.01)$ and the SHAM $(p<0.05)$ groups. The pressures exerted by the RF of the TH group were significantly higher than the ones of the Control $(p<0.001)$ and the SHAM $(p<0.01)$ groups. The pressures exerted by the LH of the TH group were significantly lower $(\mathrm{p}<0.001)$ than in the Control and the SHAM groups. The pressures exerted by the LH of the TH group at the $1^{\text {st }}(15.89 \pm 3.12 \%), 2^{\text {nd }}(23.49 \pm 2.13 \%), 3^{\text {rd }}(25.21 \pm 2.39 \%)$ and $4^{\text {th }}$ $(24.85 \pm 1.41 \%)$ weeks post-surgery were lower $(\mathrm{p}<0,001 ; \mathrm{p}<0.001 ; \mathrm{p}<0.01 ; \mathrm{p}<0.05$, respectively) than the pre-surgical value $(34.37 \pm 0.97 \%)$. The LH values of the TH group at the $1^{\text {st }}$, the $2^{\text {nd }}$, the $3^{\text {rd }}$ and the $4^{\text {th }}$ weeks post-surgery were significantly lower than the ones of both the Control $(\mathrm{p}<0,001 ; \mathrm{p}<0.001 ; \mathrm{p}<0.01$ and $\mathrm{p}<0.001$, respectively) and the SHAM $(\mathrm{p}<0,001 ; \mathrm{p}<0.01 ; \mathrm{p}<0.05$ and $\mathrm{p}<0.01$, respectively) groups.

Difference in the pressure exerted by the RH was neither observed within the TH group nor between the TH group and the other groups (Figure 4).

Pressure ratios. No difference was observed in the Control and the SHAM groups. Likewise, no difference was also found between these 2 groups. Pre-surgical ratios were similar between the 3 studied groups.

The $\mathrm{F} / \mathrm{H}$ ratios of the $\mathrm{TH}$ group were significantly superior $(\mathrm{p}<0.001)$ to the ones of the Control and the SHAM groups. The F/H ratios of the TH group at the $1^{\text {st }}(1.00 \pm 0.11), 2^{\text {nd }}$ $(0.73 \pm 0.03)$ and $3^{\text {rd }}(0.75 \pm 0.07)$ weeks post-surgery were significantly higher $(\mathrm{p}<0.001$; $\mathrm{p}<0.05$ and $\mathrm{p}<0.05$ respectively) than the pre-surgical value $(0.50 \pm 0.05)$. The $\mathrm{F} / \mathrm{H}$ ratios of the TH group at the $1^{\text {st }}$, the $2^{\text {nd }}$, the $3^{\text {rd }}$, the $4^{\text {th }}(0.64 \pm 0.04)$ and the $6^{\text {th }}(0.68 \pm 0.08)$ weeks postsurgery were greater $(\mathrm{p}<0,001 ; \mathrm{p}<0.001 ; \mathrm{p}<0.001 ; \mathrm{p}<0.05$ and $\mathrm{p}<0.05$ respectively) than the ones of the Control group. The $\mathrm{F} / \mathrm{H}$ ratios of the TH group at the $1^{\text {st }}, 2^{\text {nd }}, 3^{\text {rd }}$ and $4^{\text {th }}$ weeks were superior ( $p<0.001 ; p<0.05 ; p<0.05$ and $p<0.05$, respectively) to the ones of the SHAM group.

The LH/RH ratio of the TH group at the $1^{\text {st }}$ week post-surgery $(0.50 \pm 0.13)$ was significantly lower $(p<0.01)$ than its pre-surgical value $(1.10 \pm 0.06)$. The $\mathrm{LH} / \mathrm{RH}$ ratios of the TH group at both the $1^{\text {st }}$ and the $2^{\text {nd }}$ weeks post-surgery $(0.74 \pm 0.12)$ were lower than the ones 
of the SHAM group $\left(\mathrm{p}<0.001\right.$ and $\mathrm{p}<0.05$, respectively) while only the $1^{\text {st }}$ week post-surgery was lower than the one of the Control group $(p<0.001)$. However, no difference was observed in the TH group for the LF/RF ratios. No difference for the LF/RF ratios in the TH group was also found compared to the other groups (Figure 5).

Time spent on 4 paws. No difference in the time spent on 4 paws was observed within the Control, the SHAM and the TH groups. However, while no difference was found between the Control and the SHAM groups, the time spent on all fours in the TH group was significantly longer $(p<0.05)$ than in the Control group (Figure 6). 


\section{Discussion}

To the best of our knowledge, no previous study used the weight-bearing distribution in static position following CNS traumas as a tool to assess functional sensorimotor deficits and spontaneous recovery. The relevance of weight-bearing distribution is evaluated in the present study following stroke and spinal cord injury. The times spent on 3 paws and on 4 paws were also taken into account in order to analyse the preferred stance pattern.

\subsection{An advanced in the weight-bearing distribution measurement.}

The SWB system solely assesses hindpaws pressure of a restricted rat in an upright position, which differs from their natural posture [16]. Moreover, due to the fact that the forepaws are placed on a Plexiglas ${ }^{\circledR}$ support, their influence on the pressure exerted by the hindpaws could not be appreciated. Yet, we just found that the role of forelimbs in postural adaptation following spinal cord injury could not be neglected. In accordance to a previous study [17], the DWB seems more suitable than SWB to distinguish behavioural impairments after CNS traumas.

\subsection{Altered weight-bearing distribution following MCAO-r and TH hemisection.}

Our results indicated that MCAO-r and spinal cord injury induced different and specific postural adaptation. Severe sensorimotor impairments after MCAO-r and spinal cord injuries were respectively confirmed by the neurological score and the BBB score throughout the protocol. Indeed, the neurological score clearly indicated that rats were spontaneously left circling after MCAO-r. Furthermore, the low BBB score revealed strong locomotor deficits for the left hindlimb.

MCAO-r. The rat weight decreased after MCAO-r as previously described [18]. Some authors showed that transection of the ECA may be associated with tissue damage of the muscles of mastication affecting the capacity of eating, inducing weight loss [19]. However, other factors contribute to the observed weight loss because this phenomenon was also observed in MCAO rats without ECA damage [19]. Moreover, our observation is in accordance to previous studies indicating a minimal postoperative weight loss in SHAM group with the ECA transection. It was then postulated that the extensive corticostriatal damage was the most likely explanation of the weight loss following MCAO-r [20].

The weight loss is a general physiological state after cerebral infarction and was not just observed on the contralateral side to the lesion in our study. The weight changes seemed not to affect the body-weight distribution during static position on the four limbs. In addition, the weight of the Sham and Control animals increased during the protocol although the 
distribution of the body-weight was not changed during 8 weeks. Further studies need to explore the effect of the weight loss on the different weight distribution pattern (on two or three limbs).

The increase of the time spent on 3 paws after MCAO-r could be associated with a reduction of the left forepaw solicitation to maintain a static position. In other words, we observed, as subjective as it might be, some difficulties to move their left forelimb and therefore to use it for weight support. This phenomenon could be explained by the hemineglected contralateral limb to the cerebral lesion that was already observed following such trauma [21]. However, the pressure exerted on all fours was not disturbed after MCAO-r. Despite the fact that the occlusion was fixed at 2 hours, the upper extremity paresis may not be severe enough [22] to be detected by the DWB system. Therefore, a permanent MCAO, inducing greater sensorimotor deficits, might lead to a postural instability that could be detected with the DWB test. It should be kept in mind that the DWB system holds the potential for further developments such as optimization of the captor sensibility.

TH hemisection. Contrary to MCAO-r, the $\mathrm{T}_{10}$ hemisection induced a decrease of the left hindpaw pressure. Surprisingly, the weight was not compensated by an increase of the right hindpaw pressure, but rather by an increase of the forepaw pressure. Indeed, contrary to healthy rats that put a third of their weight onto their forepaws, spinal cord hemisected rats placed up to half of their weight. Moreover, our data seemed to indicate that the additional weight supported by the forepaws is equally divided onto the right and left sides. Contrary to the BBB test that only summarizes the notion of weight-bearing as a hindlimb plantar support and excludes the forelimb contribution, the DWB system allows quantified measure of weight-bearing pressure on each paw [15].

We also showed that the time spent on 4 paws was increased after $\mathrm{T}_{10}$ hemisection. This result might be explained by a lack of stability onto 3 paws. Moreover, the rat might not have enough force to lift its forelimbs due to their greater participation in the body weight support.

\subsection{What other behavioural tests could be used in complement to the DWB?}

The DWB test specifically brings information about postural stability in static conditions without inducing fatigue. Therefore, additional behavioural tests could be performed in order to cover a broader scope of the complex rat sensorimotor behaviour [23].

DWB is known not to provide information on gait and stride [17]. Yet, gait analysis, which brings plentiful of information about neurological diseases, could easily be coupled with the DWB test. The CatWalk is an automated gait system built upon optical technologies that could provide information about kinematic impairments after such traumas $[8,24]$. 
The DWB test could also be coupled with a balance assessment such as the beam balance task. Indeed, it is a widely used technique to assess deficits of the vestibulomotor function in different traumatic afflictions [25]. We can also proposed to associate the DWB test with the 'Inclined Plane' test that evaluates the ability of a rat to maintain its position on a tilted surface (slowly rotating the support until the animal loses grip) [26].

\subsection{Could the DWB inform us on other parameters that nociceptive information?}

Initially, the analysis of the weight-bearing distribution was used as an index of pain based on the assumption that nociceptive inputs might alter paw weight distribution [16]. Indeed, in a neuropathic pain model the decrease in paw pressure of the affected limb displayed a high degree of correlation with the well-known pain indicator, the von Frey test [27]. However, the postural control structures, located in the brainstem and cerebellum, receive different afferent inputs that are not restricted to nociceptive information [26]. Therefore, plasticity resulting from stroke and spinal cord injuries could affect several types of afferent inputs (from muscles, joints, skin) disturbing supraspinal postural networks. Moreover, the pattern of spinal postural reflexes could also be modified by stroke or spinal cord injuries [28-30]. It may contribute to cause specific changes of basic body configuration [26].

\subsection{Conclusion}

Our study showed that the DWB test should be used to assess both the severity of CNS trauma and the effectiveness of pharmacological and/or rehabilitation strategies. We strongly recommend continuing the investigation of postural instability by the use of DWB test after different CNS traumas (different stroke models or contusion, compression at cervical or thoracolumbar levels spinal cord injuries). 


\section{Acknowledgements}

This work was supported by public [Aix-Marseille Université (AMU), Centre National de la Recherche Scientifique (CNRS) and Agence Nationale de la Recherche (ANR TRAUMATISM 09-BLAN-0295-02)] and private grants [Association Libre d'Aide à Recherche sur la Moelle Epinière (ALARME), Demain Debout and Combattre la Paralysie Associations]. We are grateful to Thelma COYLE for her help in Matlab data analysis. We are grateful to Jean-Pierre GRIMALDI for his help in technical support. 


\section{References}

[1] Warlow C. Can neurologists influence stroke incidence, and do they? Journal of the Royal College of Physicians of London 1998;32:466-72.

[2] Wyndaele M, Wyndaele JJ. Incidence, prevalence and epidemiology of spinal cord injury: what learns a worldwide literature survey? Spinal cord 2006;44:523-9.

[3] Schaar KL, Brenneman MM, Savitz SI. Functional assessments in the rodent stroke model. Experimental \& translational stroke medicine 2010;2:13.

[4] Metz GA, Merkler D, Dietz V, Schwab ME, Fouad K. Efficient testing of motor function in spinal cord injured rats. Brain research 2000;883:165-77.

[5] Muir GD, Webb AA. Mini-review: assessment of behavioural recovery following spinal cord injury in rats. The European journal of neuroscience 2000;12:3079-86.

[6] Lipsanen A, Jolkkonen J. Experimental approaches to study functional recovery following cerebral ischemia. Cellular and molecular life sciences : CMLS 2011;68:3007-17.

[7] Schallert T, Fleming SM, Leasure JL, Tillerson JL, Bland ST. CNS plasticity and assessment of forelimb sensorimotor outcome in unilateral rat models of stroke, cortical ablation, parkinsonism and spinal cord injury. Neuropharmacology 2000;39:777-87.

[8] Li S, Shi Z, Zhang H, Liu X, Chen S, Jin J, et al. Assessing gait impairment after permanent middle cerebral artery occlusion in rats using an automated computer-aided control system. Behavioural brain research 2013;250:174-91.

[9] Matsuda F, Sakakima H, Yoshida Y. The effects of early exercise on brain damage and recovery after focal cerebral infarction in rats. Acta physiologica 2011;201:275-87.

[10] Virley D, Beech JS, Smart SC, Williams SC, Hodges H, Hunter AJ. A temporal MRI assessment of neuropathology after transient middle cerebral artery occlusion in the rat: correlations with behavior. Journal of cerebral blood flow and metabolism : official journal of the International Society of Cerebral Blood Flow and Metabolism 2000;20:563-82.

[11] Arvanian VL, Schnell L, Lou L, Golshani R, Hunanyan A, Ghosh A, et al. Chronic spinal hemisection in rats induces a progressive decline in transmission in uninjured fibers to motoneurons. Experimental neurology 2009;216:471-80.

[12] Longa EZ, Weinstein PR, Carlson S, Cummins R. Reversible middle cerebral artery occlusion without craniectomy in rats. Stroke; a journal of cerebral circulation 1989;20:84-91. [13] Uluc K, Miranpuri A, Kujoth GC, Akture E, Baskaya MK. Focal cerebral ischemia model by endovascular suture occlusion of the middle cerebral artery in the rat. Journal of visualized experiments : JoVE 2011.

[14] Menzies SA, Hoff JT, Betz AL. Middle cerebral artery occlusion in rats: a neurological and pathological evaluation of a reproducible model. Neurosurgery 1992;31:100-6.

[15] Basso DM, Beattie MS, Bresnahan JC. A sensitive and reliable locomotor rating scale for open field testing in rats. Journal of neurotrauma 1995;12:1-21.

[16] Bove SE, Calcaterra SL, Brooker RM, Huber CM, Guzman RE, Juneau PL, et al. Weight bearing as a measure of disease progression and efficacy of anti-inflammatory compounds in a model of monosodium iodoacetate-induced osteoarthritis. Osteoarthritis and cartilage / OARS, Osteoarthritis Research Society 2003;11:821-30.

[17] Tetreault P, Dansereau MA, Dore-Savard L, Beaudet N, Sarret P. Weight bearing evaluation in inflammatory, neuropathic and cancer chronic pain in freely moving rats. Physiology \& behavior 2011; 104:495-502.

[18] Zvejniece L, Svalbe B, Liepinsh E, Pulks E, Dambrova M. The sensorimotor and cognitive deficits in rats following 90- and 120-min transient occlusion of the middle cerebral artery. Journal of neuroscience methods 2012;208:197-204.

[19] Dittmar M, Spruss T, Schuierer G, Horn M. External carotid artery territory ischemia impairs outcome in the endovascular filament model of middle cerebral artery occlusion in rats. Stroke; a journal of cerebral circulation 2003;34:2252-7. 
[20] Virtanen T, Jolkkonen J, Sivenius J. Re: External carotid artery territory ischemia impairs outcome in the endovascular filament model of middle cerebral artery occlusion in rats. Stroke; a journal of cerebral circulation 2004;35:e9-10; author reply e9-.

[21] Reep RL, Corwin JV, Cheatwood JL, Van Vleet TM, Heilman KM, Watson RT. A rodent model for investigating the neurobiology of contralateral neglect. Cognitive and behavioral neurology : official journal of the Society for Behavioral and Cognitive Neurology 2004;17:191-4.

[22] Kleim JA, Boychuk JA, Adkins DL. Rat models of upper extremity impairment in stroke. ILAR journal / National Research Council, Institute of Laboratory Animal Resources 2007;48:374-84.

[23] Sedy J, Urdzikova L, Jendelova P, Sykova E. Methods for behavioral testing of spinal cord injured rats. Neuroscience and biobehavioral reviews 2008;32:550-80.

[24] Hamers FP, Koopmans GC, Joosten EA. CatWalk-assisted gait analysis in the assessment of spinal cord injury. Journal of neurotrauma 2006;23:537-48.

[25] Zausinger S, Hungerhuber E, Baethmann A, Reulen H, Schmid-Elsaesser R. Neurological impairment in rats after transient middle cerebral artery occlusion: a comparative study under various treatment paradigms. Brain research 2000;863:94-105.

[26] Deliagina TG, Orlovsky GN, Zelenin PV, Beloozerova IN. Neural bases of postural control. Physiology 2006;21:216-25.

[27] Vrinten DH, Hamers FF. 'CatWalk' automated quantitative gait analysis as a novel method to assess mechanical allodynia in the rat; a comparison with von Frey testing. Pain 2003;102:203-9.

[28] DeVries AC, Nelson RJ, Traystman RJ, Hurn PD. Cognitive and behavioral assessment in experimental stroke research: will it prove useful? Neuroscience and biobehavioral reviews 2001;25:325-42.

[29] Dietz V, Muller R, Colombo G. Locomotor activity in spinal man: significance of afferent input from joint and load receptors. Brain : a journal of neurology 2002;125:2626-34. [30] Lyalka VF, Orlovsky GN, Deliagina TG. Impairment of postural control in rabbits with extensive spinal lesions. Journal of neurophysiology 2009;101:1932-40. 


\section{Legend}

Figure 1: Dynamic Weight Bearing system. The device consists of a Plexiglas ${ }^{\star}$ enclosure with a calibrated weight transducer pad in which the rat is allowed to move freely. Using a synchronized video-recording and a scaled map of the stimulated captors, the pressure exerted by each paw is measured.

Figure 2: Time spent on 4 paws after MCAO-r. The time spent on all fours is expressed as a percentage of the total time spent on 3 and 4 paws. Significant differences in the time spent on 4 paws are indicated by * (MCAO pre- vs. MCAO post-), + (Control vs. MCAO), $\S$ (SHAM vs. MCAO) and \# (Control vs. SHAM). (One symbol $\mathrm{p}<0.05 ; 2$ symbols $\mathrm{p}<0.01$ and 3 symbols $\mathrm{p}<0.001)$.

Figure 3: $B B B$ locomotor rating scale. The score is plot over time after spinal cord hemisection. Significant differences in the BBB scores are indicated by * $\mathrm{TH}$ pre- $v$. TH or $+++, \mathrm{p}<0.001)$.

Figure 4: Pressure exerted by each paw after thoracic hemisection. The pressure exerted by each paw is expressed as a percentage of the total weight. A. Pressure of the left forepaw. B. Pressure of the right forepaw. C. Pressure of the left hindpaw. D. Pressure of the right hindpaw. Significant differences in the pressure exerted by each paw are indicated by * (TH pre- vs. TH post), $+($ Control vs. TH) and $\S($ SHAM vs. TH). (One symbol $\mathrm{p}<0.05 ; 2$ symbols $\mathrm{p}<0.01$ and 3 symbols $\mathrm{p}<0.001)$.

Figure 5: Pressure ratios after thoracic hemisection. A. The sum of pressure exerted by the forepaws (F) normalized by the sum of pressure exerted by the hindpaws $(\mathrm{H})$. B. The pressure exerted by the left forepaw (LF) normalized by the pressure exerted by the right forepaw (RF). C. The pressure exerted by the left hindpaw (LH) normalized by the pressure exerted by the right hindpaw $(\mathrm{RH})$. Significant differences in the pressure ratios are indicated by * $(\mathrm{TH}$ pre- vs. TH post), + (Control vs. TH) and $\S($ SHAM vs. TH). (One symbol $\mathrm{p}<0.05 ; 2$ symbols $\mathrm{p}<0.01$ and 3 symbols $\mathrm{p}<0.001)$.

Figure 6: Time spent on 4 paws after thoracic hemisection. The time spent on all fours is expressed as a percentage of the total time spent on 3 and 4 paws. 